\title{
Single breath-hold 3D LGE using stack of spiral trajectory
}

\author{
Yang Yang ${ }^{1 *}$, Peter Shaw ${ }^{3}$, Jorge A Gonzalez ${ }^{3}$, Christopher M Kramer ${ }^{3,2}$, Michael Salerno ${ }^{3,2}$ \\ From 18th Annual SCMR Scientific Sessions \\ Nice, France. 4-7 February 2015
}

\section{Background}

Late gadolinium enhancement (LGE) imaging is the gold standard for noninvasive evaluation of myocardial scar. In the clinical setting, the standard imaging protocol involves a breath-held segmented inversion-prepared 2D Cartesian acquisition at a single location. This breathhold scan is repeated 10-14 times to cover the entire left ventricle. Although multislice 2D LGE has shown great diagnostic accuracy, the multiple breath-holds increase the scan time to approximately $10 \mathrm{~min}$ and patient fatigue which can result in poor breath-holding and ghosting artifact. In this study, we propose to perform the 3D LGE imaging in a single breath-hold using a stack of spiral trajectory.

\section{Methods}

Single breath-hold 3D stack of spiral LGE images covering the entire LV were acquired in 27 subjects undergoing clinical scans following the conventional multisilce 2D LGE on a Siemens 1.5T Avanto scanner. The 3D spiral LGE sequence consisted of 12 partitions of a dual density spiral trajectory. Each spiral readout was $4 \mathrm{~ms}$ long with 24 interleaves to support $1.5 \mathrm{x}$ Nyquist in the center and $0.7 x$ Nyquist in the edge of kspace. At each cardiac cycle, 2 out of 24 interleaves were acquired for each partition resulting in an acquisition window of approximately $160 \mathrm{~ms}$. All of the spirals were acquired in a single 12 heart beat breath-hold. Other sequence parameters included: TR $7 \mathrm{~ms}$, TE $1 \mathrm{~ms}$, TI 300 400ms, FA $20^{\circ}$, FOV $340 \mathrm{~mm}$, in-plane resolution $1.5 \mathrm{~mm}, 12$ slices, and thickness $8 \mathrm{~mm}$. The images

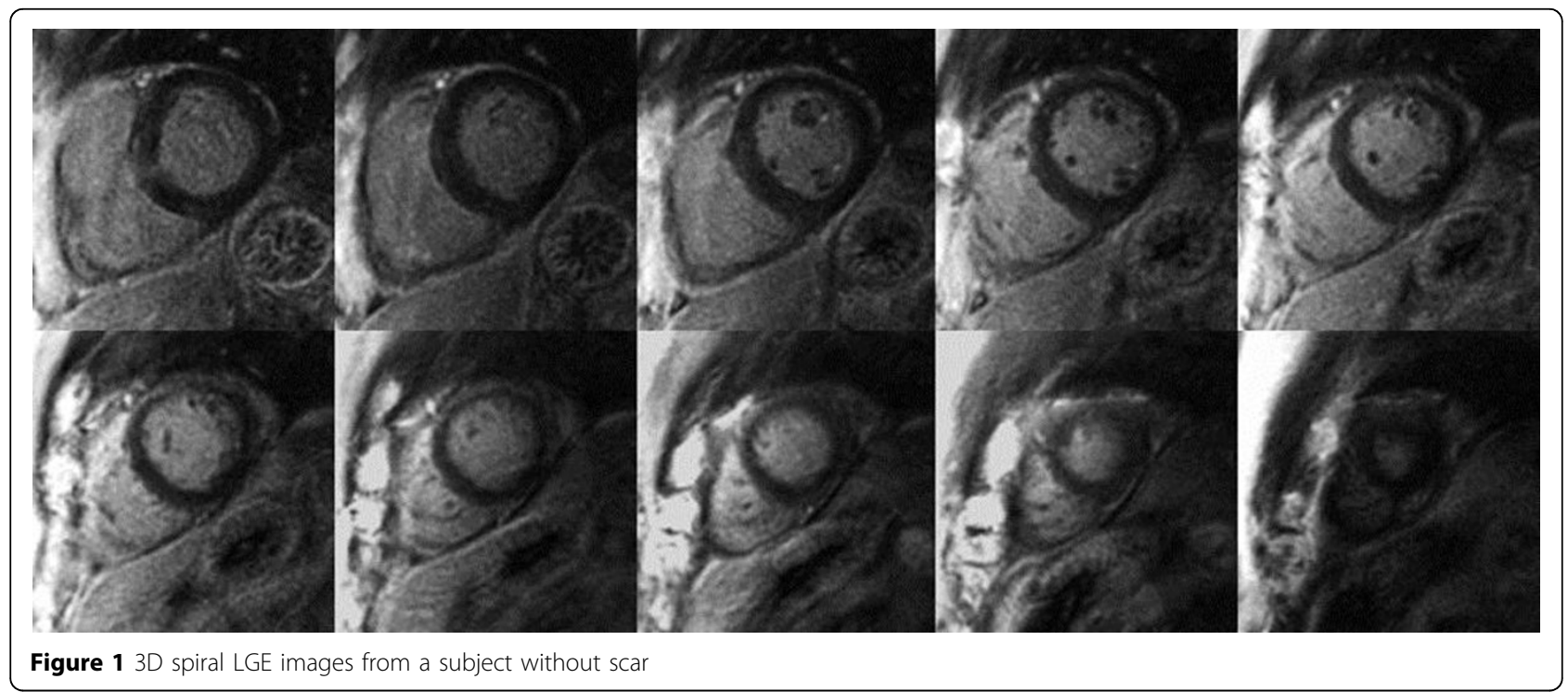

'Biomedical Engineering, University of Virginia, Charlottesville, VA, USA

Full list of author information is available at the end of the article 


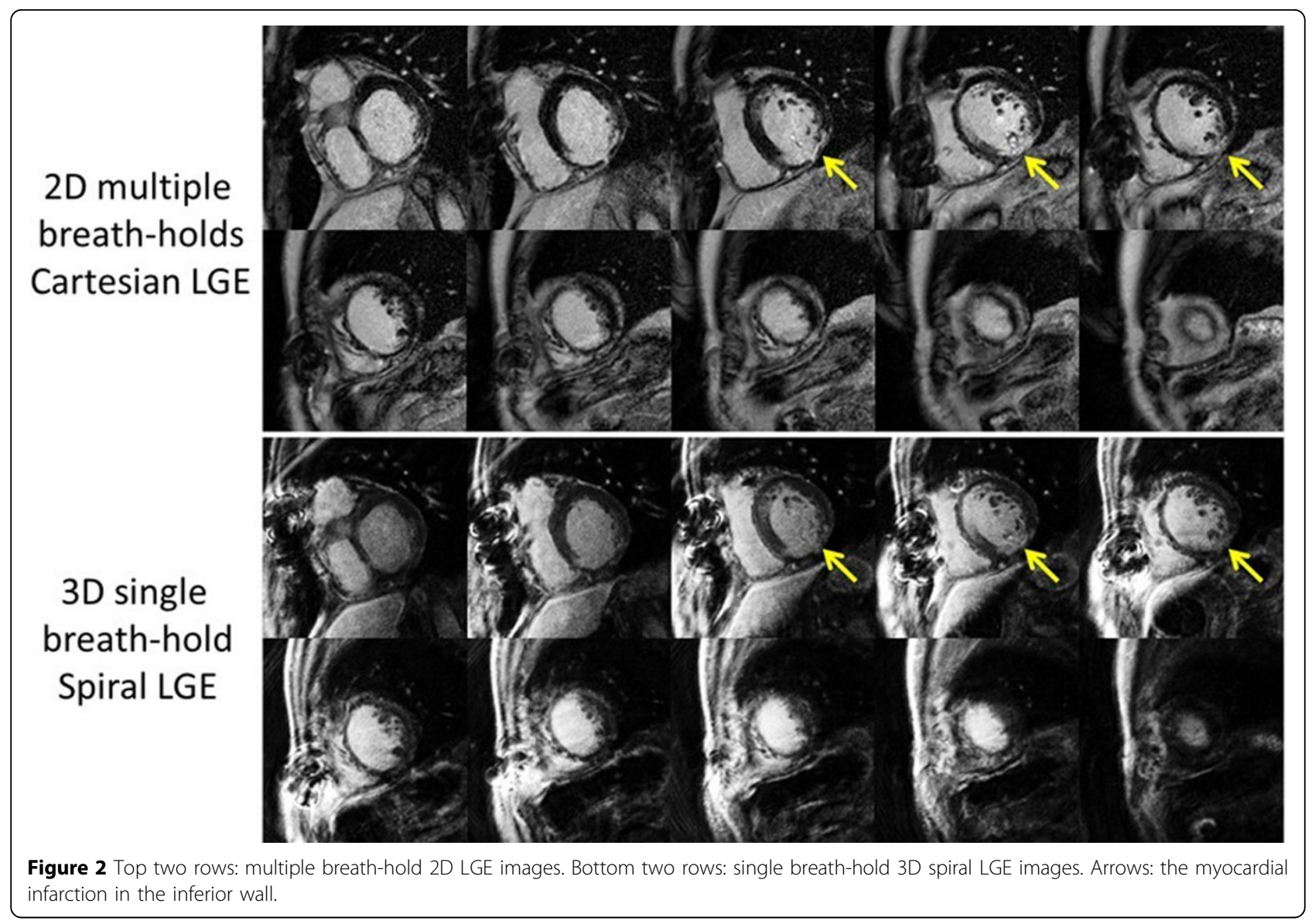

were reconstructed using SPIRiT. Image quality was rated on a 5 point scale (1- very poor to 5 excellent) by two cardiologists.

\section{Results}

Figure 1 shows the typical negative 3D LGE images from a subject without any scar. Figure 2 shows the multislice 2D LGE images (top two rows) and 3D LGE images (bottom two rows) from patients undergoing a viability study. Both of the $2 \mathrm{D}$ and $3 \mathrm{D}$ LGE images show a myocardial infarction in the inferior wall. Although the 3D LGE images were acquired within one breath-hold, the SNR of the images were still adequate for diagnostic purpose. The multislice 2D LGE scan time required approximately $10 \mathrm{~min}$ while the $3 \mathrm{D}$ LGE scan time took only $10 \mathrm{~s}$. The average image quality score was 3.7 from cardiologist 1 and 3.6 from cardiologist 2 .

\section{Conclusions}

We demonstrate the successful application of single breath-hold 3D LGE imaging using stack of spiral trajectories. As with the standard 2D multislice LGE images, 3D LGE images are able to differentiate myocardial scar, while the scan time is dramatically reduced. Such an approach will improve patient throughput in CMR.

\section{Funding}

K23 HL112910-01.

\section{Authors' details}

${ }^{1}$ Biomedical Engineering, University of Virginia, Charlottesville, VA, USA. ${ }^{2}$ Radiology, University of Virginia, Charlottesville, VA, USA. ${ }^{3}$ Medicine, University of Virginia, Charlottesville, VA, USA.

Published: 3 February 2015

doi:10.1186/1532-429X-17-S1-Q45

Cite this article as: Yang et al:: Single breath-hold 3D LGE using stack of spiral trajectory. Journal of Cardiovascular Magnetic Resonance 201517 (Suppl 1):Q45. 